Physics

Physics Research Publications

Communications: Entanglement switch for dipole arrays

Q. Wei S. Kais

Y. P. Chen

This paper is posted at Purdue e-Pubs.

http://docs.lib.purdue.edu/physics_articles/1341 


\title{
Communications: Entanglement switch for dipole arrays
}

\author{
Qi Wei, ${ }^{1}$ Sabre Kais, ${ }^{1, a)}$ and Yong P. Chen ${ }^{2}$ \\ ${ }^{1}$ Department of Chemistry and Birck Nanotechnology Center, Purdue University, West Lafayette, \\ Indiana 47907, USA \\ ${ }^{2}$ Department of Physics and Birck Nanotechnology Center, Purdue University, West Lafayette, \\ Indiana 47907, USA
}

(Received 25 January 2010; accepted 25 February 2010; published online 29 March 2010)

\begin{abstract}
We propose a new entanglement switch of qubits consisting of electric dipoles oriented along or against an external electric field and coupled by the electric dipole-dipole interaction. The pairwise entanglement can be tuned and controlled by the ratio of the Rabi frequency and the dipole-dipole coupling strength. Tuning the entanglement can be achieved for one, two, and three-dimensional arrangements of the qubits. The feasibility of building such an entanglement switch is also discussed. (C) 2010 American Institute of Physics. [doi:10.1063/1.3366522]
\end{abstract}

Entanglement is a quantum mechanical property that describes a correlation between quantum mechanical systems. It has no classical analog and has been lying in the heart of the foundation of quantum mechanics. The desire to understand, tune, and manipulate quantum entanglement is of fundamental importance in the field of quantum information and computation. ${ }^{1-4}$ Recently, we studied a set of localized spins coupled through exchange interaction and subject to an external magnetic field. ${ }^{5-8}$ We demonstrated for such a class of one-dimensional magnetic systems, that entanglement can be controlled and tuned by varying the anisotropy parameter in the Hamiltonian and by introducing impurities into the systems. ${ }^{4}$ In this letter, we propose a new entanglement switch of qubits consisting of the electric dipole moment of diatomic polar molecules, oriented along or against an external electric field and coupled by the electric dipole-dipole interaction.

Recent progress in methods for producing, trapping, and controlling cold polar molecules make them an excellent candidate for quantum computation. ${ }^{9-12}$ Trapped polar molecules was proposed as a novel physical realization of a quantum computer by a number of authors. ${ }^{12-20}$ In this proposal, the qubits are the molecular electric dipole moments which can only orient along $(|0\rangle)$ or against $(|1\rangle)$ the external electric fields. Each qubit is one polar molecule with equal spacing along the axis. For such a system, tuning and controlling the entanglement between the dipoles is of great importance.

The Hamiltonian of $N$-trapped dipoles in an external electric field reads ${ }^{21}$

$$
\mathbf{H}=\hbar \sum_{i=1}^{N} \omega_{i} \hat{S}_{i}^{z}+\hbar \sum_{i \neq j}^{N} \Omega_{i j} \hat{S}_{i}^{+} \hat{S}_{j}^{-}
$$

where $\hat{S}$ 's are the dipole operators and related to Pauli matrices, $\hat{S}_{i}^{+}$and $\hat{S}_{j}^{-}$represent dipole excitation and deexcitation, respectively. $\omega_{i}$ is the transition frequency of the

\footnotetext{
a) Author to whom correspondence should be addressed. Electronic mail: kais@purdue.edu.
}

dipole on site $i$, which is a function of dipole moment and external electric fields at site $i$.

$$
\hbar \omega_{i}=|\vec{d} \cdot \vec{E}|,
$$

where $\vec{d}$ is the electric dipole moment which we assume the same for each site. $\Omega_{i j}$ is the dipole-dipole coupling constant between sites $i$ and $j$, which is determined by the strength of dipole moment, the direction of the external electric field and the lattice constant of the dipole array.

$$
\hbar \Omega_{i j}=\frac{|\vec{d}|^{2}\left(1-3 \cos ^{2} \theta\right)}{\left|\vec{r}_{i j}\right|^{3}},
$$

where $\theta$ is the angle between $\vec{r}_{i j}$ and external electric field.

We will expand the Hamiltonian in pure standard basis, $\{|00\rangle,|01\rangle,|10\rangle,|11\rangle\}$, and all the eigenstates will be obtained by diagonalizing the Hamiltonian matrix. For example, for $N=2$, we obtained the following four eigenvectors: $\quad \Psi_{1}=|00\rangle, \quad\left|\Psi_{2}=(1 / \sqrt{2})(|01\rangle-|10\rangle), \quad\right| \Psi_{3}=(1 / \sqrt{2})$ $\times(|01\rangle+|10\rangle), \quad \Psi_{4}=|11\rangle$ with corresponding eigenvalues: $E_{1}=0, E_{2}=\omega-\Omega, E_{3}=\omega+\Omega, E_{4}=2 \omega$. For the ratio $\omega / \Omega$ $<1, \Psi_{2}$ is fully entangled ground state. However, for $\omega / \Omega>1$ the nonentangled state $\Psi_{1}$ is the ground state. Thus, we have a curve crossing at $\omega / \Omega=1$.

The concept of entanglement of formation is related to the amount of entanglement needed to prepare the state $\rho$, where $\rho$ is the density matrix. It was shown by Wootters ${ }^{22}$ that concurrence is a good measure of entanglement. ${ }^{23}$ The concurrence $C$ is given by ${ }^{22}$

$$
C(\rho)=\max \left\{0, \lambda_{1}-\lambda_{2}-\lambda_{3}-\lambda_{4}\right\} .
$$

For a general state of two qubits, $\lambda_{i}$ 's are the eigenvalues, in decreasing order, of the Hermitian matrix $R \equiv \sqrt{\sqrt{\rho}} \widetilde{\rho} \sqrt{\rho}$ where $\rho$ is the density matrix and $\tilde{\rho}$ is the spin-flipped state defined as

$$
\widetilde{\rho}=\left(\sigma_{y} \otimes \sigma_{y}\right) \rho^{*}\left(\sigma_{y} \otimes \sigma_{y}\right),
$$

where the $\rho^{*}$ is the complex conjugate of $\rho$ and is taken in the standard basis, which for a pair of two level particles is $\{|00\rangle,|01\rangle,|10\rangle,|11\rangle\}$. 

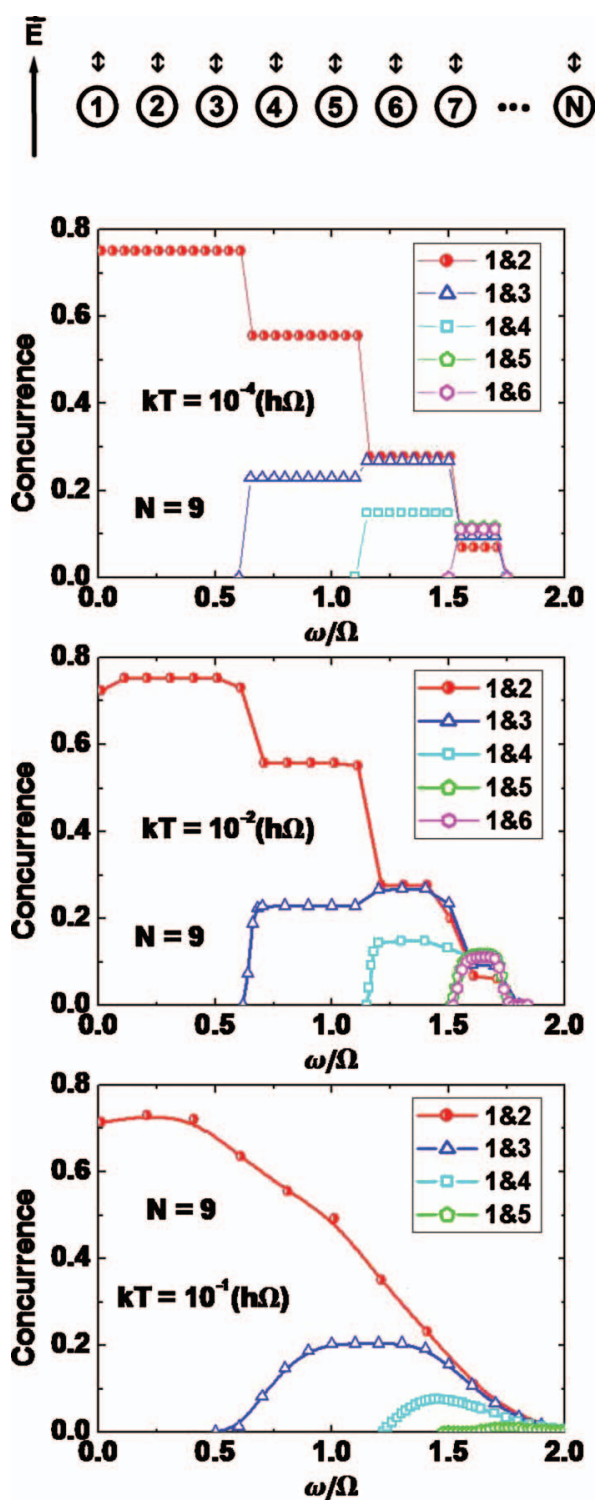

FIG. 1. Pairwise concurrence [Eq. (4)] of one-dimensional arrangements of the dipoles as one varies the ratio $\omega / \Omega$ at $k T=10^{-4}, 10^{-2}$, and $10^{-1} \hbar \Omega$, respectively, for $N=9$ dipoles.

In order to calculate thermal entanglement, we need the temperature dependent density matrix and the density matrix for a system in equilibrium at a temperature $T$ reads: $\rho=e^{-\beta H / Z}$ with $\beta=1 / k T$ and $Z$ is the partition function, $Z=\operatorname{Tr}\left(e^{-\beta \hat{H}}\right)$. In this case, the partition function is

$$
Z(T)=\sum_{i} g_{i} e^{-\beta \lambda_{i}}
$$

where $\lambda_{i}$ is the $i$ th eigenvalue and $g_{i}$ is the degeneracy. And the corresponding density matrix can be written

$$
\rho(T)=\frac{1}{Z} \sum_{i}^{N} e^{-\beta \lambda_{i}}\left|\Phi_{i}\right\rangle\left\langle\Phi_{i}\right|,
$$

where $\left|\Phi_{i}\right\rangle$ is the $i$ th eigenfunction. For pairwise thermal entanglement, we can get reduced density matrix as a function of temperature in the same way, which leads to temperature dependent entanglement.

In Fig. 1 we show the tuning of the pairwise entangle-
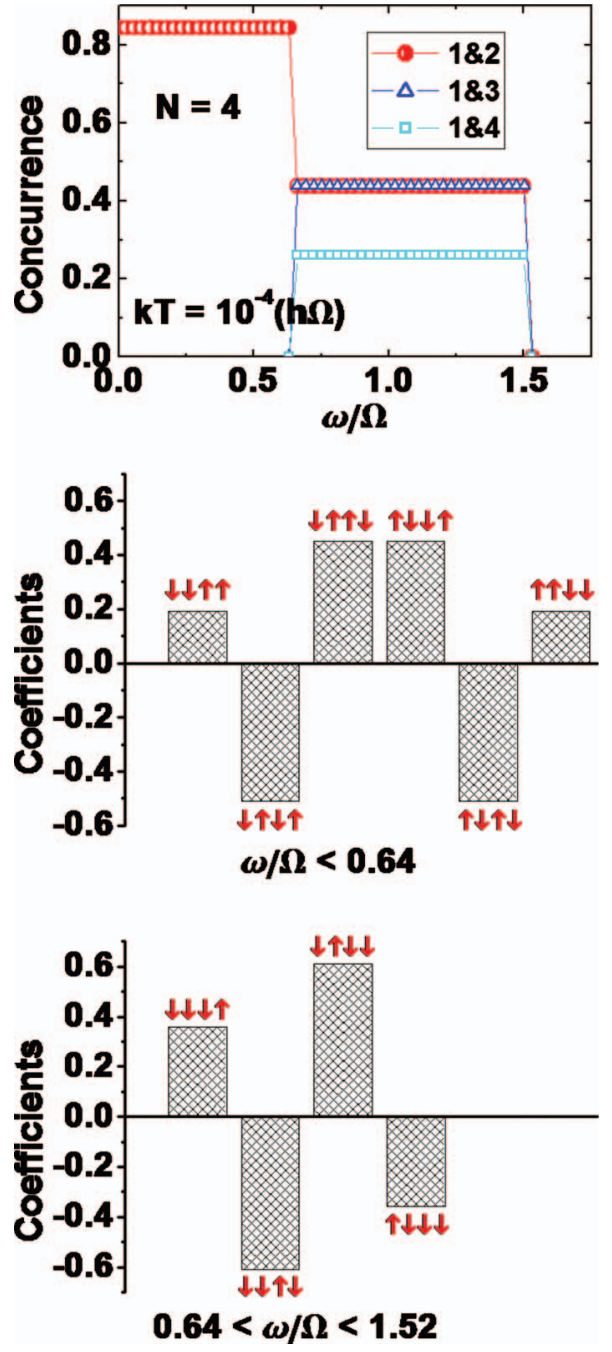

FIG. 2. Pairwise concurrence [Eq. (4)] and coefficients of the wave function of one-dimensional arrangements of the dipoles as one varies the ratio $\omega / \Omega$ at $k T=10^{-4} \hbar \Omega$ for $N=4$ dipoles.

ment, measured by concurrence, of one-dimensional arrangements of the dipoles as one varies the ratio $\omega / \Omega$ at different temperatures for $N=9$ dipoles. Here we took all transition frequencies to be the same, $\omega_{i}=\omega$, and the nearest neighbor dipole-dipole interaction to be the same, $\Omega_{i, i+1}=\Omega_{i, i-1}=\Omega$. All other dipole-dipole coupling constants $\Omega_{i, j \neq i \pm 1}$ can be expressed in terms of $\Omega$. Thus, we have two parameters to vary, the ratio $\omega / \Omega$ and temperature $k T$. At $k T \sim 0$ one has a constant entanglement over a long ratio $\omega / \Omega$ and sharp transitions or jumps to lower values at other values of $\omega / \Omega$. It is worth mentioning that for $\omega / \Omega<0.634$, entanglement is only between dipoles 1 and 2 . For $0.634<\omega / \Omega<1.14$ dipole one becomes entangled also with dipole 3 and with other dipoles until we reach $\omega / \Omega \sim 1.74$, above this value the concurrence is zero between all sites. As one increases the temperature, the curve becomes smoother as mixing occur with higher states. Calculations for $N=3,4, \ldots 8$ gave similar behavior of tuning and controlling entanglement as for the case $N=9$. To show how the populations changes at the transition point, we present in Fig. 2 the coefficients of the wave function for $N=4$ before and after the transition point $\omega / \Omega=0.64$. When $\omega / \Omega<0.64$, the ground state wave 

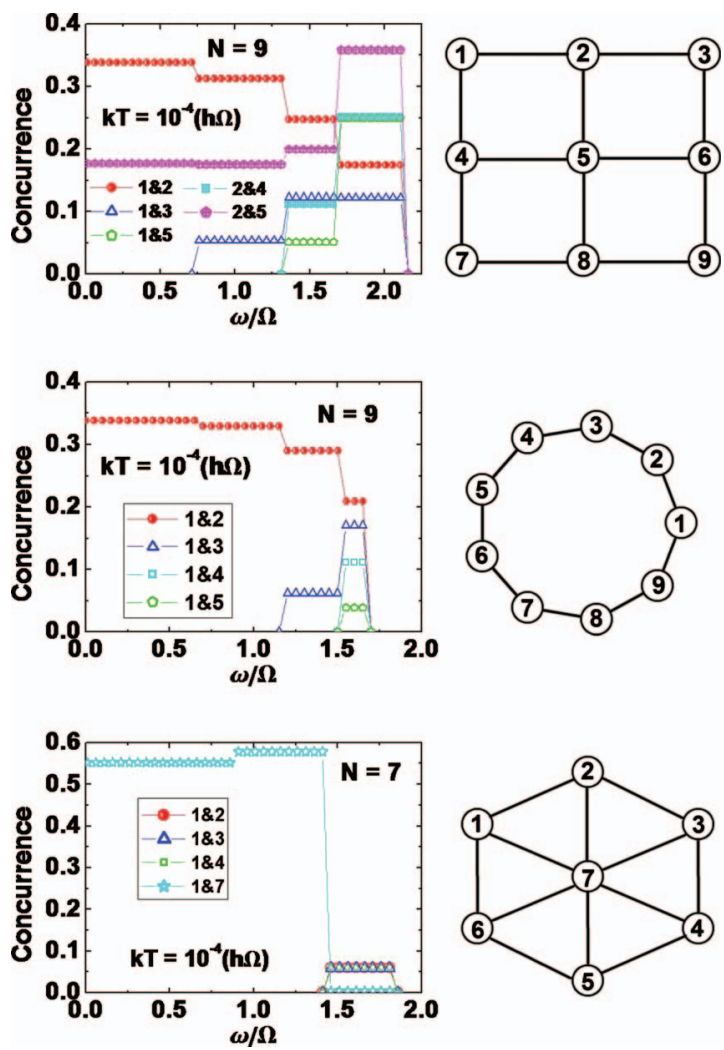

FIG. 3. Pairwise concurrence [Eq. (4)] for different two-dimensional arrangements of the dipoles as one varies the ratio $\omega / \Omega$ at $k T=10^{-4} \hbar \Omega$. The external electric field is perpendicular to the $2-d$ plane.

function is $\quad|\Psi\rangle=0.19|\downarrow \downarrow \uparrow \uparrow\rangle-0.51|\downarrow \uparrow \downarrow \uparrow\rangle+0.45|\downarrow \uparrow \uparrow \downarrow\rangle$ $+0.45|\uparrow \downarrow \downarrow \uparrow\rangle-0.51|\uparrow \downarrow \uparrow \downarrow\rangle+0.19|\uparrow \uparrow \downarrow \downarrow\rangle$, however, when $\omega / \Omega>0.64$, the ground state wave function becomes $|\Psi\rangle$ $=0.36|\downarrow \downarrow \downarrow \uparrow\rangle-0.61|\downarrow \downarrow \uparrow \downarrow\rangle+0.61|\downarrow \uparrow \downarrow \downarrow\rangle-0.36|\uparrow \downarrow \downarrow \downarrow\rangle$. Figures 3 and 4 show a similar phenomena for two and threedimensional arrangements of the dipoles. The pairwise entanglement decreases as one increases the dimensionality of the system and the temperature.

There have been rapid advances made recently in cooling, trapping and manipulating atomic (Rydberg) $)^{24-26}$ and molecular dipoles. ${ }^{10,11,27-29}$ For example, a wide variety of ground state polar molecules with large electric dipole moments (several debyes, where $1 \mathrm{D} \sim 3.3 \times 10^{-30} \mathrm{C} \mathrm{m}$ ) have been cooled to ultracold ( $\sim \mathrm{mK}$ or below) regime, some even near quantum degeneracy. ${ }^{29}$ These developments provide exciting opportunities to experimentally realize the entanglement switch described above, which requires tuning $\omega / \Omega$
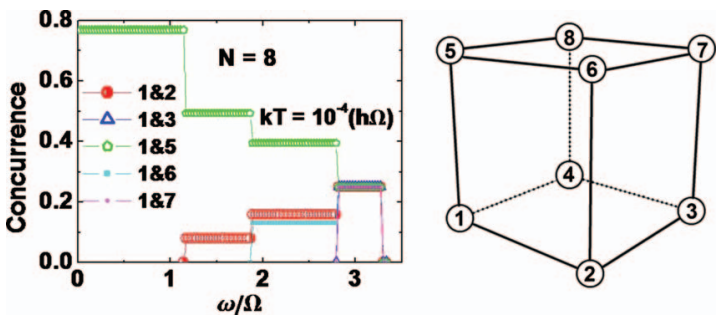

FIG. 4. Pairwise concurrence [Eq. (4)] of three-dimensional simple cubic arrangements of the dipoles as one varies the ratio $\omega / \Omega$ at $k T=10^{-4} \hbar \Omega$. Here $\Omega$ is the dipole-dipole coupling constant between dipole 1 and dipole $2, \Omega=\Omega_{12}$. The external electric field is along dipoles 1 to 5 . around 1 , and cooling the dipoles to temperatures $(T)$ corresponding to a fraction of the dipole-dipole interaction energy $(\Omega) \cdot{ }^{30}$ For a dipole moment $(p)$ of few debyes, and a typical experimental electric field $\left(\sim 10^{5} \mathrm{~V} / \mathrm{m}\right.$, required for the dipole moment to actually manifest itself $\left.{ }^{12,31}\right)$, dipoledipole separation $(d)$ on the order of $10 \mathrm{~nm}$ is required for $\omega / \Omega \sim 1$. Such $d$ is much shorter than what can be achieved in typical optical lattices (as envisaged in Ref. 12 which corresponds to the regime of $\omega / \Omega \gg 1$ ), but can be realized with arrays of nanoscale plasmon-enhanced electric/electrooptical traps recently proposed ${ }^{32,33}$ (where extremely tight, few-nanometer confinement, and trap frequencies exceeding $100 \mathrm{MHz}$ are shown to be possible). At such short $d$, the dipole-dipole interaction $\Omega=p^{2} / 4 \pi \epsilon_{0} d^{3}$ is on the order of $0.1 \mathrm{~K}$. Cooling to a small fraction of such a temperature scale is easily within the current experimental technology. Furthermore, $\omega / \Omega$ can be tuned experimentally by the electrical field, and/or by $d$ (e.g., by varying the microtrap configuration).

In summary, we presented a new way to construct an entanglement switch in an optical lattice and discussed the experimental feasibility of building such switch. The realization of such a scheme will have a profound impact on the implementation of quantum gates in quantum computing with trapped polar molecules. The similar idea could in principle also be applied to other systems with electric dipoles, such as quantum dots and excitons in nanostructures.

We would like to thank the ARO for financial support. Y.P.C. acknowledges partial support from National Science Foundation (Grant CCF No. 0829918).

${ }^{1}$ M. A. Nielsen and I. L. Chuang, Quantum Computation and Quantum Information (University Press, Cambridge, 2000).

${ }^{2}$ L. Amico, R. Fazio, A. Osterloh, and V. Vedral, Rev. Mod. Phys. 80, 517 (2008).

${ }^{3}$ V. Vedral, Nature (London) 453, 1004 (2008).

${ }^{4}$ S. Kais, Adv. Chem. Phys. 134, 493 (2007).

${ }^{5}$ O. Osenda, Z. Huang, and S. Kais, Phys. Rev. A 67, 062321 (2003).

${ }^{6}$ Z. Huang, O. Osenda, and S. Kais, Phys. Lett. A 322, 137 (2004).

${ }^{7}$ Z. Huang and S. Kais, Int. J. Quantum Inf. 3, 483 (2005).

${ }^{8}$ Z. Huang and S. Kais, Phys. Rev. A 73, 022339 (2006).

${ }^{9}$ R. Krems, W. C. Stwalley, and B. Friedrich, Cold Molecules: Theory, Experiment, Applications (Taylor Francis, London, 2009).

${ }^{10}$ D. Herschbach, Faraday Discuss. 142, 9 (2009).

${ }^{11}$ B. Friedrich and J. M. Doyle, ChemPhysChem 10, 604 (2009).

${ }^{12}$ D. DeMille, Phys. Rev. Lett. 88, 067901 (2002).

${ }^{13}$ G. K. Brennen, I. H. Deutsch, and P. S. Jessen, Phys. Rev. A 61, 062309 (2000).

${ }^{14}$ G. K. Brennen, C. M. Caves, P. S. Jessen, and I. H. Deutsch, Phys. Rev. Lett. 82, 1060 (1999).

${ }^{15}$ A. Micheli, G. K. Brennen, and P. Zoller, Nat. Phys. 2, 341 (2006).

${ }^{16}$ M. D. Lukin, M. Fleischhauer, R. Cote, L. M. Duan, D. Jaksch, J. I. Cirac, and P. Zoller, Phys. Rev. Lett. 87, 037901 (2001).

${ }^{17}$ D. Jaksch, J. I. Cirac, and P. Zoller, Phys. Rev. Lett. 85, 2208 (2000).

${ }^{18}$ S. F. Yelin, K. Kirby, and R. Cote, Phys. Rev. A 74, 050301(R) (2006).

${ }^{19}$ E. Charron, P. Milman, A. Keller, and O. Atabek, Phys. Rev. A 75, 033414 (2007).

${ }^{20}$ E. Kuznetsova, R. Cote, K. Kirby, and S. F. Yelin, Phys. Rev. A 78, 012313 (2008).

${ }^{21}$ Z. Ficek and S. Swain, Quantum Interference and Coherence Theory and Experiment (Springer, New York, 2004).

${ }^{22}$ W. K. Wootters, Phys. Rev. Lett. 80, 2245 (1998). 
${ }^{23}$ S. Hill and W. K. Wootters, Phys. Rev. Lett. 78, 5022 (1997).

${ }^{24}$ E. Urban, T. A. Johnson, T. Henage, L. Isenhower, D. D. Yavuz, T. G. Walker, and M. Saffman, Nat. Phys. 5, 110 (2009).

${ }^{25}$ T. Amthor, C. Giese, C. S. Hofmann, and M. Weidemuller, Phys. Rev. Lett. 104, 013001 (2010).

${ }^{26}$ R. Heidemann, U. Raitzsch, V. Bendkowsky, B. Butscher, R. Low, and T. Pfau, Phys. Rev. Lett. 100, 033601 (2008)

${ }^{27}$ K. K. Ni, S. Ospelkaus, M. H. G. de Miranda, A. Pe'er, B. Neyenhuis, J. J. Zirbel, S. Kotochigova, P. S. Julienne, D. S. Jin, and J. Ye, Science 322, 231 (2008).
${ }^{28}$ J. Deiglmayr, A. Grochola, M. Repp, K. Mörtlbauer, C. Glück, J. Lange, O. Dulieu, R. Wester, and M. Weidermüller, Phys. Rev. Lett. 101, 133004 (2008).

${ }^{29}$ L. D. Carr, D. DeMille, R. V. Krems and J. Ye, New J. Phys. 11, 055049 (2009).

${ }^{30}$ M. Aymar and O. Dulieua, J. Chem. Phys. 122, 204302 (2005).

${ }^{31}$ J. L. Bohn, e-print arXiv:0901.0276.

${ }^{32}$ D. E. Chang, J. D. Thompson, H. Park, V. Vuletić, A. S. Zibrov, P. Zoller, and M. D. Lukin, Phys. Rev. Lett. 103, 123004 (2009).

${ }^{33}$ B. Murphy and L. V. Hau, Phys. Rev. Lett. 102, 033003 (2009). 